\title{
Patient understanding of, satisfaction with, and perceived utility of whole-genome sequencing: findings from the MedSeq Project
}

\author{
J. Scott Roberts, $\mathrm{PhD}^{1}$, Jill O. Robinson, $\mathrm{MA}^{2}$, Pamela M. Diamond, $\mathrm{PhD}^{3}$, Archana Bharadwaj, $\mathrm{MPH}^{1}$, \\ Kurt D. Christensen, $\mathrm{PhD}^{4}$, Kaitlyn B. Lee, $\mathrm{BA}^{2}$, Robert C. Green, MD, $\mathrm{MPH}^{4}$ and \\ Amy L. McGuire, PhD, JD²; for the MedSeq Project team
}

Purpose: To examine patients' experiences with clinical use of whole-genome sequencing (WGS).

Methods: A randomized trial compared primary care and cardiology patients receiving WGS and family health history (FH) information or FH information alone. 202 patients were surveyed before (BL) and up to 6 months after disclosure of results $(6 \mathrm{M})$

Results: Patients (mean age $=55$ years; $50 \%$ female; $81 \%$ college graduates) reported low levels of decisional regret (mean: 7.1/100) and high satisfaction with physicians' disclosure of results (median: 29/30). Compared with the FH-only arm, patients receiving WGS results were more likely to report learning accurate disease risk information (odds ratio $=7.45$ ) and findings influential for medical treatment (odds ratio $=2.39$ ). Sessions where WGS results were disclosed took longer (30 vs. 15 minutes), particularly for primary care patients. Patients' expected utility of sequencing at BL was higher than perceived utility at $6 \mathrm{M}$ in several domains, including impacting medical decision making ( $87 \%$ vs. $54 \%)$ and influencing medication choice ( $73 \%$ vs. $32 \%)$.

Conclusion: Patients were satisfied with their physicians' communication of WGS results and perceived them as medically useful. Discrepancies in expected versus perceived utility of WGS results suggest a need to temper patients' expectations about its potential benefits.

Genet Med advance online publication 4 January 2018

Key Words: cardiology; informed consent; patient education; patient satisfaction; primary care

\section{INTRODUCTION}

Whole-genome and whole-exome sequencing (WGS/WES) are increasingly being used in medical practice for clinical indications spanning diagnostic testing, predisposition screening for rare variants associated with Mendelian conditions, and susceptibility testing for common diseases. ${ }^{1}$ The potential to tailor prevention and treatment based on a patient's genomic information has generated significant enthusiasm among proponents of precision medicine. ${ }^{2}$ Yet the use of WGS in the clinical setting poses significant challenges in the translation and communication of WGS/WES results, with informed consent and return of results as two processes that may be particularly complicated. ${ }^{3}$ Not only is sequencing analysis inherently complex due to the volume and ambiguity of information generated, but patients (and many providers) may lack the genetic and health literacy skills required to adequately comprehend and act upon recommendations stemming from test results. ${ }^{4}$

To date, much attention has been focused on the challenges of informed consent in WGS/WES, including ethical and practical dilemmas posed by the management and disclosure of potential incidental (or secondary) findings. ${ }^{5}$ However, relatively few empirical studies have examined the experience of medical patients undergoing WGS/WES, with these studies primarily focused on cancer patients in particular (e.g., ref. 6). Studies are needed in a broader range of medical contexts to identify both barriers and best practices for informed consent and return of WGS/WES results. In this paper, we report on findings from a randomized clinical trial examining the process and impact of using WGS in cardiology and primary care settings. More specifically, we examine patients' understanding of informed consent for WGS, their satisfaction with their physicians' disclosure of test results, and their perceptions of the utility of their individual WGS findings. By gauging patient experiences and responses to implementation of WGS/WES, we hope to shed light on some of the practical and ethical challenges involved in translating genome sequencing from research into clinical practice.

\section{MATERIALS AND METHODS}

Participants and procedures

The MedSeq Project utilizes a randomized controlled trial design to compare provision of family health history (FH)

\footnotetext{
${ }^{1}$ Department of Health Behavior and Health Education, University of Michigan School of Public Health, Ann Arbor, Michigan, USA; ${ }^{2}$ Center for Medical Ethics and Health Policy, Baylor College of Medicine, Houston, Texas, USA; ${ }^{3}$ Division of Health Promotion \& Behavioral Sciences, University of Texas Houston School of Public Health, Houston, Texas, USA; ${ }^{4}$ Division of Genetics, Department of Medicine, Brigham and Women's Hospital and Harvard Medical School, Boston, Massachusetts, USA. Correspondence: J. Scott Roberts (jscottr@umich.edu)
} 
information alone with family history plus WGS (FH + WGS) in two clinical contexts: primary care and cardiology. Details of the trial methodology have been published elsewhere. ${ }^{7}$ In brief, both cardiologists specializing in cardiomyopathy $(n=7)$ and primary care physicians $(n=9)$ were recruited for the study from an academic network of outpatient practices in Boston, Massachusetts. Each provider recruited interested and eligible patients from their practices (mean: 12.4 patients ( $\mathrm{SD}=7.7$ ) per provider; range: $2-28$ patients for cardiologists, 4-16 patients for primary care physicians). Interested patient-participants were then referred to study coordinators, who confirmed eligibility and obtained informed consent.

Patients in the primary care trial were healthy adults aged 41-68 years at the time of enrollment. Patients in the cardiology trial (age 18 and over at enrollment) had diagnoses of hypertrophic cardiomyopathy or dilated cardiomyopathy, with prior or concurrent targeted genetic testing for their condition. All patients underwent a structured family history assessment. In addition, half randomly underwent WGS and had the results interpreted on a report sent to their physician; genomic findings of significance reported to study physicians included monogenic disease risk secondary findings (mean = 0.3 per primary care patient vs. 0.2 per cardiology patient), carrier findings (mean $=2.7$ findings per primary care patient vs. 1.9 per cardiology patient), and variants related to the patient's current condition (mean $=0.6$ findings per patient in the cardiology cohort only). A results disclosure session involved patient-physician discussion of either findings of the family history assessment alone $(\mathrm{FH})$, or family history results along with the WGS report $(\mathrm{FH}+\mathrm{WGS})$. Prior to disclosure visits, physicians were provided access to a study Genome Resource Center staffed by a medical geneticist and genetic counselor who could answer questions about family history reports, genome sequencing reports, and/or study procedures.

Overall, 205 patient-participants provided informed consent. At the baseline visit, 203 patient-participants completed study surveys and online family history assessment, underwent a blood draw for genetic analyses, and were randomized to either the WGS or control arm. Once sequencing results and a family health history summary were generated, study staff scheduled a visit to discuss the results. At this visit, patients learned whether they were randomized to receive WGS. The physician discussed family history findings and, for those randomized to receive WGS, the Genome Report as well. Together, the physician and patient made clinical care management decisions based on the findings. Patientparticipants were surveyed immediately after the disclosure visit, as well as 6 weeks and 6 months following disclosure.

\section{Measures}

Patient-participant characteristics

Patient-participant demographic characteristics were collected via self-report and included age, gender, race/ethnicity, annual household income, and highest education level. A full listing of the measures utilized in this project has been presented elsewhere. ${ }^{7}$ Genetic literacy was assessed using a genomic sequencing knowledge scale developed in the ClinSeq sequencing study. ${ }^{8}$ This measure includes 11 items assessing knowledge of genomic sequencing benefits and limitations (e.g., "Even if a person has a variant in a gene that affects their risk of a disease, they may not develop that disease"). Numeracy was measured using the Subjective Numeracy Scale, a validated 8-item measure that uses patient selfassessment to distinguish numeracy skill levels in an efficient manner. ${ }^{9}$

\section{Understanding of informed consent}

To measure understanding of the study's informed consent process, patient-participants completed a novel 21-item true/ false quiz during the baseline visit. Items assessed domains including the study's purpose, procedures, risks and benefits, and policies regarding return of sequencing results. Items answered correctly were summed to yield a possible score from 0 to 21 .

\section{Perceived understanding}

Patient-participants were assessed regarding how well they believed they understood key study information at two time points. At baseline, their perceived understanding of key elements of the study's informed consent process was assessed using three items adapted from a validated measure of the quality of informed consent. ${ }^{10}$ Subjective understanding was also assessed with two items at the results disclosure session. One assessed how well participants believed they understood the new information they had just learned, while the other assessed how well participants believed they needed to understand the information to make health-care decisions $(1=$ not at all to $5=$ extremely well $)$.

\section{Satisfaction with communication of study results}

Immediately following their results disclosure session, participants were asked to rate their satisfaction with how test results (i.e., FH only or FH + WGS) were communicated by their study physician. Five items (e.g., "How well was your doctor able to explain new information and its meaning in a way that you could understand?") were administered, with response choices on a 6-point scale $(1=$ not at all to $6=$ very much). Reponses were summed to create an overall satisfaction score (range: 5 to 30, with higher scores indicating higher levels of satisfaction). This measure demonstrated a high level of reliability in this study (Cronbach's alpha = 0.85). In addition, one item asked participants to rate the overall amount of information they had just learned ("too much" versus "too little" versus "just right"), and one item assessed how much new information their results had yielded ("none" versus "some" versus "a lot").

\section{Perceived utility and decisional regret}

At the study's baseline visit, participants were asked whether they thought that study results would influence different aspects 
of their health care (e.g., medications, advance planning). Six items were administered, with response choices on a 4-point scale (no, probably no, probably yes, yes). An item was considered to be endorsed as an expected study benefit if the response was "probably yes" or "yes." These items were also administered at the 6-month follow-up visit, where participants were asked about the extent to which study results actually had influenced their health care (response choices: not at all, slightly, moderately, very much, extremely). An item was considered to be endorsed as an achieved study benefit if the response was "siightly" or "greater." At the study's 6-week visit, a validated measure assessing the extent to which respondents experienced regret following a health-related decision was administered. ${ }^{11}$ Possible scores ranged from 0 to 100 , with higher scores indicating higher levels of decisional regret. Items were anchored to the decision to participate in the MedSeq Project.

\section{Data analyses}

Descriptive statistics characterized the sample in terms of its demographic characteristics and responses on questionnaire items. Chi-square, Wilcoxon rank-sum, and $t$-test analyses compared group differences (e.g., cardiology versus primary care) on individual survey items. Multiple regression analyses were performed on three composite study outcome measures: informed consent knowledge (linear regression examining knowledge scores at baseline), satisfaction with communication (linear regression examining satisfaction scores after results disclosure), and decisional regret (logistic regression examining regret scores at 6 weeks following results disclosure). In addition, regression analyses examined differences between the FH + WGS and FH-only arms on perceived utility items assessed at 6 months. Because some outcome variable scores were highly skewed, transformations were required. For example, decisional regret was transformed into a dichotomous variable, with logistic regression used to identify significant correlates. All regression analyses included the following variables: age, gender, race, education, genetic literacy, numeracy, study cohort (cardiology versus primary care), and randomization arm. Statistical calculations were performed using SPSS statistical software (IBM Corporation, Armonk, NY).

\section{Demographics}

\section{RESULTS}

A total of 202 patient-participants provided data, approximately half from primary care and half from cardiology. The mean age of the sample was 55 years, with an even split between male and female (although the primary care cohort was more likely to be female and the cardiology cohort more likely to be male). Most participants were non-Hispanic white $(87 \%)$, had completed college $(81 \%)$, and had an annual household income exceeding $\$ 100,000$ (63.9\%). There were no significant differences in demographic characteristics between the randomization arms, except that within the primary care cohort, WGS arm participants were more likely to have graduated college ( $94 \%$ vs. $78 \%, P<0.05)$. Further details on sample characteristics are presented in Table 1.

\section{Understanding of informed consent}

Participant responses on the understanding of informed consent measure are summarized in Table 2. The overall mean score on this measure was 18.9 out of 21 items (90\%) correct, and mean scores on this measure did not differ significantly by randomization arm or study cohort. Overall,

Table 1 Sample characteristics $(n=202)$

\begin{tabular}{|c|c|c|c|c|c|}
\hline \multirow{2}{*}{$\begin{array}{l}\text { No. }(\%) \text {, unless } \\
\text { otherwise noted }\end{array}$} & \multicolumn{2}{|c|}{ Cardiology cohort $(n=102)$} & \multicolumn{2}{|c|}{ Primary care cohort $(n=100)$} & \multirow{2}{*}{$\begin{array}{l}\text { Total sample } \\
(n=202)\end{array}$} \\
\hline & WGS + FH $(n=50)$ & FH-only $(n=52)$ & WGS + FH $(n=50)$ & FH-only $(n=50)$ & \\
\hline Mean age, in years (SD) & $55.9(16.1)$ & $55.9(12.2)$ & $55.2(7.0)$ & $54.6(7.6)$ & $55.3(11.3)$ \\
\hline \multicolumn{6}{|l|}{ Gender $r^{a}$} \\
\hline Female & $24(48 \%)$ & $19(36.5 \%)$ & $28(56 \%)$ & $30(60 \%)$ & $101(50 \%)$ \\
\hline Male & $26(52 \%)$ & $33(63.5 \%)$ & $22(44 \%)$ & $20(40 \%)$ & $101(50 \%)$ \\
\hline \multicolumn{6}{|l|}{ Race/Ethnicity } \\
\hline Non-Hispanic white & $43(86 \%)$ & $46(88.5 \%)$ & $44(88 \%)$ & $43(86 \%)$ & $176(87.1 \%)$ \\
\hline Other & $7(14 \%)$ & $6(11.5 \%)$ & $6(12 \%)$ & $7(14 \%)$ & $26(12.9 \%)$ \\
\hline \multicolumn{6}{|l|}{ Annual household income } \\
\hline$\$ 0-\$ 34,999$ & $11(22.9 \%)$ & $5(10 \%)$ & $2(4.1 \%)$ & $4(8.5 \%)$ & $22(11.3 \%)$ \\
\hline$\$ 35,000-\$ 99,999$ & $14(29.2 \%)$ & $15(30 \%)$ & $7(14.3 \%)$ & $12(25.5 \%)$ & $48(24.7 \%)$ \\
\hline
\end{tabular}

$\mathrm{FH}$, family history; WGS, whole-genome sequencing.

Eight participants did not report household income.

a Primary care cohort more likely than cardiology cohort to be female, $P<0.05$. 'bithin primary care cohort, WGS $+\mathrm{FH}$ arm more likely than FH-only arm to be college graduate, $P<0.05$. 
Table 2 Understanding of informed consent Survey item (correct answer) \% Correct

\begin{tabular}{l}
$\begin{array}{l}\text { Study purpose (What are the main reasons this project is being } \\
\text { done?) }\end{array}$ \\
$\begin{array}{l}\text { To study how genomic information is used in health-care } \\
\text { decisions (True) }\end{array}$ \\
$\begin{array}{l}\text { To test a new gene-targeting drug (False) } \\
\text { To create a map of the human genome (False) }\end{array}$ \\
$\begin{array}{l}\text { Study procedures (This study involves...) } \\
\text { An experimental drug (False) }\end{array}$ \\
$\begin{array}{l}\text { A blood draw (True) } \\
\text { At least } 4 \text { study visits (True) }\end{array}$ \\
$\begin{array}{l}\text { Completing surveys (True) } \\
\text { Genetic testing of my family members (False) }\end{array}$ \\
$\begin{array}{l}\text { Randomization (What information will you receive as part of } \\
\text { this study?) }\end{array}$ \\
$\begin{array}{l}\text { I have a } 50 \% \text { chance of receiving info from my whole } \\
\text { genome sequence (True) }\end{array}$ \\
\hline
\end{tabular}

I will definitely receive information from my whole genome $\quad 94 \%$ sequence (False)

I have a $50 \%$ chance of receiving info about my family $\quad 70 \%$

history (False)

I will definitely receive information about my family history $\quad 60 \%$

(True)

Results returned (As part of this study, I may receive results that include...)

My risk of developing certain diseases for which there are no known preventions, cures, or treatments available

(True)

Personal genetic traits, such as eye and hair color (False)

New information that doctors or researchers do not

understand (True)

Study procedures and related risks

My results will be placed in my medical record (True)

$100 \%$

My results will be discarded when I complete this study

$98 \%$

(False)

Partners HealthCare System will pay for additional medical

tests to follow up on my results (False)

I'm protected by federal law from genetic discrimination by

$92 \%$

health insurers and employers (True)

My results will not be accessible to anyone outside of

Partners HealthCare System (False)

My de-identified WGS results will be shared with other

researchers in a national database over the Internet (True)

WGS, whole-genome sequencing.

$63 \%$ of the patient-participants answered at least $90 \%$ of items correctly, with only $3 \%$ of participants answering less than $70 \%$ of items correctly. The vast majority ( $>97 \%)$ knew the study's core procedures (e.g., multiple study visits, survey completion), understood that the main purpose of the project was to study how genomic information is used in health-care decisions, and recognized they could potentially receive genetic risk information about diseases for which there were no available treatments. Two of the items most frequently answered incorrectly concerned study randomization procedures, with $40 \%$ of participants not recognizing that they would receive family history results regardless of the study arm to which they were randomized. A subset of participants also appeared to hold misconceptions regarding types of results to be returned to them, with $19 \%$ incorrectly responding that they would not receive information that doctors themselves do not understand. All participants knew that study results would be placed in their medical record, but $14 \%$ did not recognize that their WGS results could be shared with outside researchers, despite the study consent form explaining that their genomic data would be deposited into the National Institutes of Health-managed database of Genotypes and Phenotypes (dbGaP).

\section{Perceived understanding}

The vast majority of participants endorsed all survey items asking about their perceived understanding of the study's informed consent process. Ninety-eight percent of patientparticipants agreed that they had made an informed choice in participating in this study, with $70 \%$ indicating that they strongly agreed. Ninety-eight percent agreed that they knew the risks of the study, with $69 \%$ strongly agreeing. Participants also generally reported high levels of perceived understanding of study results communicated to them in the disclosure visit (e.g., $86 \%$ indicated that they understood "well" or "extremely well" any new information they had learned) but there were slight differences observed by randomization arm, with participants in the FH + WGS arm less likely than those in the FH-only arm to report they understood new information "extremely well" $(26 \%$ vs. $51 \%, P<0.01)$. Only $72 \%$, however, indicated that they needed to understand the information they discussed with their doctor "well" or "extremely well" in order to make health-care decisions.

\section{Satisfaction with communication of results}

As shown in Table 3, participants rated satisfaction with their physician's communication of study results very highly; the median overall score was $29 / 30$, with no individual provider receiving less than a median score of 27/30 from his or her study patients. There were significant randomization arm differences on two survey items, with WGS + FH arm participants' ratings lower than FH-only participants' ratings on "How satisfied are you with how well you understand the information you just discussed with your doctor?" (64\% vs. $78 \%$ endorsed "very much," $P<0.05)$ and "How confident are you that you could fully explain the meaning of the information you just discussed to a family member?" ( $42 \%$ vs. $75 \%$ endorsed "very much," $P<0.001$ ).

There were also significant randomization arm differences in terms of the amount of information participants reported receiving from their study results. Participants in the FH + WGS arm were much more likely than those in the FH-only arm to report having received a lot of new information from study results $(51 \%$ vs. $5 \%, P<0.001)$, 
Table 3 Findings from results disclosure sessions

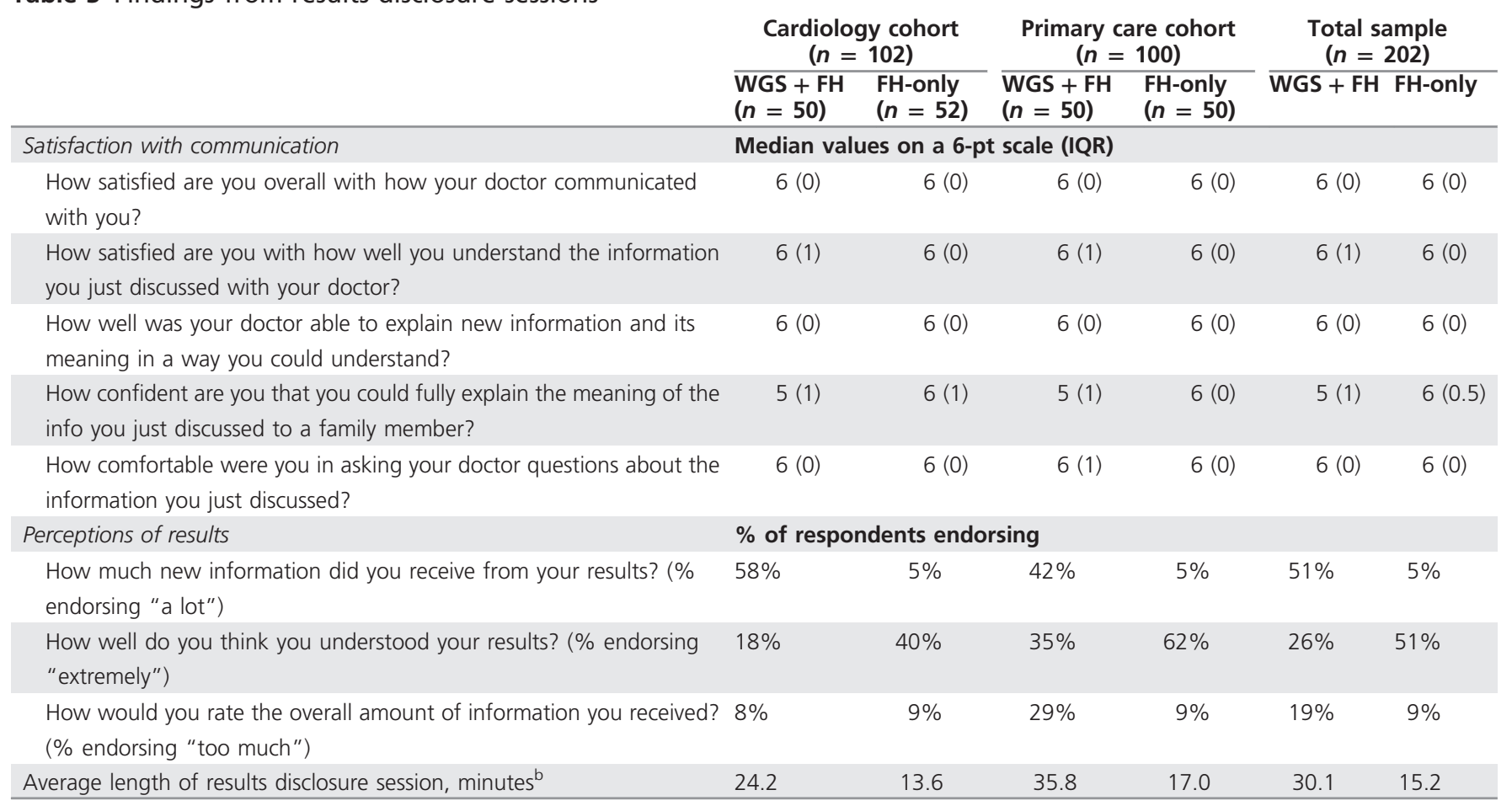

FH, family history; IQR, interquartile range; WGS, whole-genome sequencing.

a Primary care cohort participants more likely to endorse "too much" information, $P<0.05$. 'Primary care sessions longer than cardiology; WGS arm longer than FH-only arm; $P<0.001$.

and they were more likely to report receiving "too much" information $(19 \%$ vs. $9 \%, P<0.05)$. Overall, primary care patients were more likely than their cardiology counterparts to report receiving "too much" information following WGS $(21 \%$ vs. $8 \%, P<0.05)$. The average length of results disclosure sessions differed both by randomization arm and study cohort. Sessions where WGS +FH results were disclosed were significantly longer than $\mathrm{FH}$-only sessions (mean $=30.1$ vs. 15.2 minutes, $P<0.001$ ), and primary care results disclosure sessions were longer overall than cardiology sessions (mean $=26.5$ vs. 18.7 minutes, $P<0.001$ ).

\section{Decisional regret}

Participants in the FH-only arm reported on average higher levels of decisional regret than participants in the WGS + FH arm (mean $=17.8$ vs. 7.1, $P<0.001$ ). Participants in the WGS + FH arm reported low overall levels of decisional regret, with a median score of 0 and a $95 \%$ confidence interval for the mean score $=4.0-10.2$ on a $0-100$ scale (with 100 being the highest possible level of decisional regret).

\section{Expected and perceived utility}

At baseline (prior to learning to which study arm they would be randomized), a majority of participants endorsed numerous expected benefits from receiving their study results: results to influence medical decision making $(86.9 \%)$, results to influence current or future medical treatment (85.1\%), accurate identification of disease risks (79.8\%), and results to influence choice of medications (73\%). At 6-month follow-up, notably fewer participants endorsed having actually achieved study benefits than had expected them at the baseline visit (see Table 4). The only exception was that $80.5 \%$ reported at least some level of benefit at 6-month follow-up regarding accurate identification of disease risk, approximately the same proportion that had reported expecting this benefit at baseline. Logistic regression analyses showed that as compared with participants in the family history arm, those in the FH + WGS arm were significantly more likely at 6 months to report that study results had (i) led to accurate identification of disease risks (odds ratio $=7.45,95 \%$ confidence interval $=2.9-19.4$ ), and (ii) influenced current medical treatment (odds ratio $=2.39,95 \%$ confidence interval $=1.2-4.6$ ).

\section{Multivariate analyses}

Table 5 summarizes regression analyses of variables associated with key study outcome measures of informed consent knowledge, decisional regret, and satisfaction with communication. These analyses showed several variables to be significantly associated with higher levels of informed consent knowledge: higher education level, higher genetic literacy, and higher subjective numeracy. The decisional regret analysis showed that participants in the FH arm were more likely than those in the FH + WGS arm to report at least some level of decisional regret. No variables were found to be significant predictors of satisfaction with communication. 
Table 4 Expected versus perceived utility of results Survey item \% Expecting (BL) \% Endorsing (6M)

FH + WGS arm odds of endorsing at $6 \mathrm{M}$ (vs. FH-only arm) ${ }^{\mathrm{a}}$

\begin{tabular}{|c|c|c|c|}
\hline Accurate identification of disease risks ${ }^{b}$ & 79.8 & 80.5 & $7.45(95 \% \mathrm{Cl}=2.9-19.4)$ \\
\hline Influence current/future medical treatment ${ }^{\mathrm{b}}$ & 85.1 & 68.1 & $2.39(95 \% \mathrm{Cl}=1.2-4.6)$ \\
\hline Influence medical decision making & 86.9 & 54.5 & $1.51(95 \% \mathrm{Cl}=0.8-2.8)$ \\
\hline Influence patient or their child's reproductive decision making & 32.5 & 18.8 & $2.11(95 \% \mathrm{Cl}=0.95-4.67)$ \\
\hline Influence choice of medications & 73.0 & 32.3 & $1.0(95 \% \mathrm{Cl}=0.5-1.9)$ \\
\hline Influence end-of-life planning & 46.0 & 25.1 & $1.0(95 \% \mathrm{Cl}=0.5-2.0)$ \\
\hline
\end{tabular}

$\%$ Expecting are those who responded Probably Yes or Yes; \% Endorsing are those who responded at least Slightly.

$6 \mathrm{M}, 6$ months after disclosure of results; BL, before disclosure of results; Cl, confidence interval; FH, family history; WGS, whole-genome sequencing.

${ }^{a}$ Analyses reported in this column controlled for patient age, gender, education, race/ethnicity, genetic literacy, and study cohort (cardiology vs. primary care). ${ }^{\mathrm{b}} \mathrm{FH}+\mathrm{WGS}$ arm more likely to endorse at $6 \mathrm{M}$ vs. FH-only arm, $P<0.05$.

Bold values denote statistically significant results.

Table 5 Summary of regression analyses

\begin{tabular}{|c|c|c|c|c|c|c|c|c|c|}
\hline Predictors & \multicolumn{3}{|c|}{ Informed consent knowledge } & \multicolumn{3}{|c|}{ Decisional regret } & \multicolumn{3}{|c|}{ Satisfaction with communication } \\
\hline Gender & 0.706 & 0.261 & 0.07 & 0.873 & $0.40,1.90$ & 0.73 & 0.367 & 0.417 & 0.38 \\
\hline Education & 0.748 & 0.348 & 0.03 & 0.984 & $0.33,2.97$ & 0.98 & -0.009 & 0.556 & 0.99 \\
\hline Genetic literacy & 0.337 & 0.113 & 0.03 & 0.945 & $0.68,1.31$ & 0.74 & 0.048 & 0.166 & 0.77 \\
\hline Randomization arm (WGS + FH vs. FH-only) & -0.041 & 0.244 & 0.87 & 0.207 & $0.10,0.43$ & $<0.001$ & -0.106 & 0.392 & 0.79 \\
\hline
\end{tabular}

$\mathrm{B}$, beta; FH, family history; OR, odds ratio; SE, standard error; WGS, whole-genome sequencing.

Multiple linear regression models used for informed consent knowledge and satisfaction with communication analyses; logistic regression model used for decisional regret analyses.

Bold values denote statistically significant results.

\section{DISCUSSION}

With use of genomic sequencing increasing, examining the implementation of WGS in clinical settings is critical to guide future practice. Existing studies have largely focused on use of WGS in cancer treatment, thereby creating a need to examine WGS in other clinical contexts. We report here on the first randomized controlled trial examining the use of WGS in cardiology and primary care settings. Overall, patientparticipants generally understood key facts about the sequencing trial, with those receiving WGS results reporting minimal decisional regret, high levels of satisfaction with their physicians' communication of sequencing results, and significant medical utility of WGS findings. However, their pretest expectations of the clinical benefits of sequencing were not fully realized.

Our findings of high levels of both objective and perceived understanding of informed consent were encouraging. Patients showed good comprehension of most key facts about the study, including its purpose, procedures, potential risks, and policies regarding return of study results. These findings differ from many previous studies that have found widespread participant misunderstandings of informed consent processes in genomics research in particular ${ }^{12}$ and clinical research more generally. ${ }^{13}$ Such findings can be explained by numerous factors. First, the patient sample was well-educated overall, with generally high levels of genetic and health literacy. Second, the study employed informed consent procedures consistent with best practices in the field. ${ }^{14}$ These included use of clear statements and plain language in the written consent form, use of expert and highly trained study research staff, and a consent process involving elicitation of participant understanding and a chance to ask questions of study personnel. There were some limitations to participants' informed consent knowledge, however. Scores were higher among those with higher levels of education, genetic literacy, and numeracy. In studies involving populations with lower health literacy, accommodations may need to be made to the consent process to ensure optimal understanding; prior work has suggested the use of enhanced consent forms, informational videos or brochures, and extended discussions as strategies to improve comprehension..$^{15}$ In additional, there were particular areas where participants were more likely to show deficits in understanding, including study randomization procedures, results to be returned, and study policies regarding data sharing. More explicit attention to these specific areas during the consent process, along with the provision of simplified consent forms summarizing study policies and procedures, may be useful to address these challenges.

Another key study finding was the high level of satisfaction participants reported with their physicians' communication of sequencing results. Patients believed that their physicians 
communicated WGS results in a clear and effective manner and felt comfortable in posing questions to them about their results. These findings are encouraging given that strong physician-patient communication has long been associated with important outcomes including satisfaction with health care and emotional health. ${ }^{16}$ Few studies have examined physician-patient communication in the context of genomic medicine, however, and our findings represent an early contribution to this emerging body of work. Physicianpatient communication in this study was likely enhanced by patient-participants' already existing relationships with their study provider. In addition, all physicians in the study received considerable genomics education (two 1-hour group classes and 4 hours of online modules) prior to disclosing study results. Finally, physicians were offered individualized support by medical genetics and genetic counselors through both telephone and e-mail. Such training and support probably prepared physicians to successfully navigate the process of discussing and conveying genome sequencing and family history results to patients.

There were notable differences in study outcomes between participants who received genome sequencing results and those who did not. Sessions where WGS results were disclosed were twice as long as $\mathrm{FH}$-only sessions, with patients who received WGS results more likely to note that they had received "too much" information and less likely to report that they understood this information very well. This finding suggests a need to simplify how sequencing results are presented to patients, as WGS may be particularly prone to creating an "information overload" experience for patients. ${ }^{3}$ Patients who received only family history results were more likely to report at least some regret about their decision to take part in the study. This finding is likely attributable to participants' disappointment about not being randomized to receive personal genomic results, which presumably held appeal to participants and was the major rationale for the study. It should be noted, however, that overall levels of decisional regret were quite low across both study arms. Participants who received their own sequencing information were much more likely to report that study results had provided new information with some level of personal or clinical utility. For example, sequencing arm participants were over seven times more likely to report that study results had yielded accurate identification of disease risk and more than twice as likely to report that results had or would influence their medical treatment. Taken together, these findings suggest that patient-participants found significant value in their WGS results. However, it should also be noted that participants expected a higher level of benefits from their study results than was actually achieved. For example, widely held expectations that results would influence medical decision making and choice of medications were often not fulfilled. Such expectations are not surprising given the amount of publicity genomic sequencing has received in the news media and the enthusiasm expressed by many scientific leaders about its future promise. ${ }^{2,17}$ Our findings suggest a need to manage patient expectations about the likelihood of clinical benefit from current approaches to WGS to achieve optimally informed medical decision making.

Although the cardiology and primary care patient cohorts were similar in terms of their high levels of informed consent knowledge and satisfaction with physician communication, there were notable differences between the groups' experience of the study results disclosure session. For example, primary care patient-participants were more likely than their cardiology counterparts to report receiving "too much" information from their study results. This finding may be due to results disclosure sessions in the primary care cohort being on average over $40 \%$ longer (26.5 vs. 18.5 minutes) than cardiology sessions. It may be that cardiologists in the study, who had greater prior experience in ordering and conveying genetic test results, were more able to efficiently communicate sequencing and family history results to participants. Indeed, the broader literature suggests that primary care physicians often lack experience and do not feel prepared for discussions of personal genomic information with patients, ${ }^{18,19}$ which may help account for these findings.

Our findings should be interpreted in light of several study limitations. There were significant selection biases with regard to both study patients and physicians, with a relatively small number of physicians participating (i.e., seven cardiologists, nine primary care physicians). These limitations make it difficult to know how study results would or would not generalize to other populations (e.g., patients with greater racial/ethnic and socioeconomic diversity; physicians at community-based hospitals). Lack of social and racial/ethnic diversity in study populations, including ours, has been a perennial problem in genomics research ${ }^{20}$ and clinical research more generally, ${ }^{21}$ and addressing this issue should be a high priority in future translational genomics research. To accelerate progress in this area, the National Human Genome Research Institute has mandated the oversampling of underrepresented minorities as a condition of funding in one of its clinical sequencing consortia. ${ }^{22}$ Another study limitation concerns the measures used for assessment. Many survey measures, including the informed consent knowledge scale, consisted of novel items and have not been formally validated. In addition, some measures (e.g., satisfaction with communication) may have been prone to social desirability biases in responses. Finally, key outcome measures such as satisfaction with communication and decisional regret had very limited variability in response, constraining the ability to detect group differences.

In conclusion, this study suggests that the integration of clinical sequencing into primary care and cardiology can be performed in a manner that promotes informed consent and effective patient-physician communication and results in high levels of patient satisfaction and perceived utility of sequencing results. Patients may overrate the likelihood of clinical benefits from sequencing, suggesting a need to temper expectations when deciding on its use in medical practice. Future research in this area with larger and more diverse 
patient populations is needed to further guide the clinical implementation of sequencing.

\section{ACKNOWLEDGMENTS}

This work was supported by National Institutes of Health grants U01 HG006500, UM1 HG006508, and K01 HG009173.

\section{DISCLOSURE}

R.C.G. receives compensation for speaking or consultation from AIA, GenePeeks, Helix, Ohana, Prudential and Veritas, and is cofounder of, adviser to, and equity holder in Genome Medical. The other authors declare no conflict of interest.

\section{REFERENCES}

1. Krier JB, Kalia SS, Green RC. Genomic sequencing in clinical practice: applications, challenges, and opportunities. Dialogues Clin Neurosci 2016;18:299-312.

2. Collins FS, Varmus $\mathrm{H}$. A new initiative on precision medicine. $N$ Engl J Med 2015;372:793-5.

3. Berg JS, Khoury MJ, Evans JP. Deploying whole genome sequencing in clinical practice and public health: meeting the challenge one bin at a time. Genet Med 2011;13:499-504.

4. Syurina EV, Brankovic I, Probst-Hensch N, Brand A. Genome-based health literacy: a new challenge for public health genomics. Public Health Genomics 2011;14:201-10.

5. Appelbaum PS, Parens E, Waldman CR, et al. Models of consent to return of incidental findings in genomic research. Hastings Cent Rep 2014;44: 22-32

6. Scollon S, Bergstrom K, Kerstein RA, et al. Obtaining informed consent for clinical tumor and germline exome sequencing of newly diagnosed childhood cancer patients. Genome Med 2014;6:69.

7. Vassy JL, Lautenbach DM, McLaughlin HM, et al. The MedSeq Project: a randomized trial of integrating whole genome sequencing into clinical medicine. Trials 2014;15:85-97.
8. Kaphingst KA, Facio FM, Cheng MR, et al. Effects of informed consent for individual genome sequencing on relevant knowledge. Clin Genet 2012;82:408-15.

9. Fagerlin A, Zikmund-Fisher BJ, Ubel PA, Jankovic A, Derry HA, Smith DM. Measuring numeracy without a math test: development of the Subjective Numeracy Scale. Med Decis Making 2007;27:672-80.

10. Joffe S, Cook EF, Cleary PD, Clark JW, Weeks JC. Quality of informed consent: a new measure of understanding among research subjects. J Natl Cancer Inst 2001;93:139-47.

11. Brehaut JC, O'Connor AM, Wood TJ, et al. Validation of a decision regret scale. Med Decis Making 2003;23:281-92.

12. Beskow LM. Lessons from HeLa cells: the ethics and policy of biospecimens. Annu Rev Genomics Hum Genet 2016;17:395-417.

13. Montalvo W, Larson E. Participant comprehension of research for which they volunteer: a systematic review. J Nurs Scholarsh 2014;46:423-31.

14. McGuire AL, Beskow LM. Informed consent in genomics and genetic research. Annu Rev Genomics Hum Genet 2010;11:361-81.

15. Nishimura A, Carey J, Erwin PJ, Tilburt JC, Murad MH, McCormick JB. Improving understanding in the research informed consent process: a systematic review of 54 interventions tested in randomized control trials. BMC Med Ethics 2013;14:28.

16. Stewart MA. Effective physician-patient communication and health outcomes: a review. CMAJ 1995:152:1423-33.

17. Caulfield T, Condit C. Science and the sources of hype. Public Health Genomics 2012;15:209-17.

18. Nippert I, Harris HJ, Julian-Reynier C, et al. Confidence of primary care physicians in their ability to carry out basic medical genetic tasks-a European survey in five countries-Part 1. J Community Genet 2011;2: $1-11$.

19. Mikat-Stevens NA, Larson IA, Tarini BA. Primary-care providers' perceived barriers to integration of genetics services: a systematic review of the literature. Genet Med 2015;17:169-76.

20. Popejoy AB, Fullerton SM. Genomics is failing on diversity. Nature 2016;538:161-4.

21. Oh SS, Galanter J, Thakur N, et al. Diversity in clinical and biomedical research: a promise yet to be fulfilled. PLOS Med 2015;12:e1001918.

22. Clinical Sequencing Evidence-Generating Research (CSER2). https:// grants.nih.gov/grants/guide/rfa-files/RFA-HG-16-010.html. Accessed 12 July 2017. 